\title{
Sandra I. McCoy: leveraging elements of games to improve sexual health
}

Received: 29 November 2018; Accepted: 03 December 2018; Published: 14 December 2018.

doi: $10.21037 /$ mhealth.2018.12.03

View this article at: http://dx.doi.org/10.21037/mhealth.2018.12.03

\section{Editor's note}

HIV is one of world's most serious public health challenges. In the US alone, there are more than 1.1 million people living with this deadly disease, and 1 out of 7 of these people are not aware of their infection. Approximately 39,800 people were diagnosed with HIV in the US in 2016. Gay, bisexual, and men who have sex with men bear a disproportionate burden by risk group (1). mHealth interventions have thus arisen with an aim to improve people's engagement in desirable sexual health services and behaviors like HIV testing, pre-exposure prophylaxis (PrEP), treatment of sexually transmitted diseases (STDs), and linkage to antiretroviral therapy (ART).

Prof. Sandra I. McCoy from the University of California, Berkeley School of Public Health and her team recently developed an innovative mHealth intervention using gamification (the use of game elements in non-game setting). The intervention, Stick To It, aims to motivate young men who have sex with men (YMSM) to engage in desirable sexual behaviors in a context of fun and ultimately increasing repeat HIV screening (2). In the following interview with Prof. McCoy, $m$ Health will invite you to have a closer look at her research in this regard.

\section{Expert introduction}

Sandra I. McCoy, PhD, MPH, is an Associate Professor of Epidemiology at the University of California, Berkeley School of Public Health. She is also a Faculty Affiliate at I4Y (Innovations for Youth) at the School of Public Health, University of California; an Affiliate Researcher at the Consortium for Research on HIV, AIDS, \& TB (CISIDAT), Cuernavaca; and a Faculty Affiliate at the Center for Effective Global Action (CEGA) at the University of California (Figure 1).

Prof. McCoy's research is focused on prevention and control of HIV and other sexually transmitted infections, demand creation for HIV prevention, treatment, and

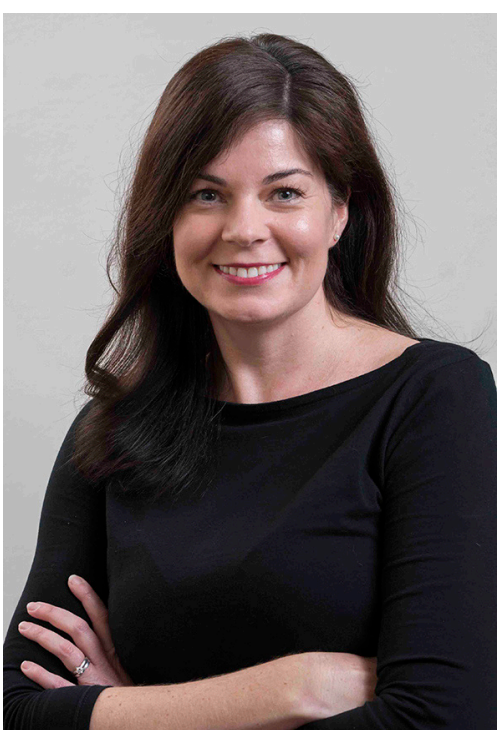

Figure 1 Prof. Sandra I. McCoy.

care programs, women's economic empowerment, multisectoral approaches to health, and impact evaluation and implementation science. She is actively involved in multiple academic societies, including International AIDS Society, International Society for Infectious Diseases, California Public Health Association-North, and Society for Epidemiologic Research.

\section{Interview}

mHealth: Having conducted research in the area of HIV prevention and control for years, what do you think are the critical issues facing the field right now?

Prof. McCoy: It's an exciting time in the field of HIV prevention and control. When I started out as a graduate student about a decade ago, the outlook was pretty dismal; there weren't many effective prevention tools that held promise to curb a rapidly growing epidemic. However, today we're living in a kind of renaissance with more 
strategies than ever at our disposal. ART is both lifesaving treatment and a strong and effective prevention tool through 'treatment as prevention' and PrEP. Treatment delivery programs form the backbone of a combination approach to epidemic control which is enhanced with effective strategies for vulnerable populations, including voluntary medical male circumcision, HIV self-testing, economic support, and behavioral approaches to support uptake and use of other programs and services. There is growing consensus that the tools to end the epidemic now exist, and the conversation has shifted to the once-impossible idea of 'getting to zero'.

The primary challenge for HIV professionals has now become one of implementation. Although the strategies mentioned above have demonstrated effectiveness in research settings (e.g., clinical trials), we need to figure out how to bring these solutions and technologies to the people who need them. This requires a concerted effort to align funding streams, policy environments, supply chains, and technical know-how. The goal of this kind of research, commonly known as 'implementation science', is to close the "know-do gap"- the delay between the discovery of effective ways to reduce mortality and morbidity and the application of these proven interventions on a wide scale.

\section{mHealth: Your team strives to use innovative methods for behavioral changes in the aspects of sexual and reproductive bealth. Can you tell us a bit more about these methods?}

Prof. McCoy: Let's use HIV as an illustrative example. Although numerous HIV prevention tools have been developed in the past decade, nearly all require a behavioral component to maximize effectiveness. For example, prevention strategies such as PrEP, the use of antiretroviral drugs to prevent HIV infection), require users to locate a provider, attend regular medical visits, adhere to the drug, be regularly screened for HIV/sexually transmitted infections (STIs) and monitored for side effects, and use condoms or other methods to avoid other STDs. Even condoms require correct and consistent use to be effective. Ultimately, this means that people must be highly motivated to maximize the effectiveness of whichever prevention strategy suits them best.

Traditionally, public health professionals have relied on strategies using 'information, education, and communication' to motivate people to engage in beneficial behaviors or services. However, these methods often erroneously assume that information is sufficient to change behavior. Such approaches primarily rely on individuals' innate desire to remain healthy; however, they ignore the fact that decisions in the real world are influenced by emotions, contexts, and systems as well as decision-making shortcuts that are often outside of conscious awareness.

The field of behavioral science leverages people's systematic biases and heuristics to positively change behaviors. These approaches use tools from behavioral economics and psychology to subtly influence behavior and include financial and in-kind incentives, social influence, commitments, and reminders. Although these strategies have been increasingly utilized to promote weight loss, smoking cessation, and the adoption of other health behaviors, there's been fewer applications to sexual and reproductive health. My team believes these tools hold great promise for HIV and STDs, including bolstering HIV testing and adherence to ART, increasing access to contraception, and stimulating demand for other HIV and STD prevention and care services.

\section{mHealth: Your team has led a pilot study using gamification intervention to increase HIV screening among young men who have sex with men in California. How did you come up with this idea in the first place?}

Prof. McCoy: The idea originated about five years ago from a colleague, Dr. Raluca Buzdugan, who noticed how the private sector was incorporating elements of games into professional settings to enhance motivation, productivity, and ultimately, the 'bottom line'. Shortly thereafter, 'gamification'-the application of game elements in non-game settings-began popping up everywhere. For example, it's now common to use smartphone apps track your physical activity to compete with friends, coffee shop or supermarket customer loyalty programs, and 'badges' for providing high-quality reviews on websites like TripAdvisor. This is likely happening because the evidence suggests that gamification works. Specifically, it creatively improves attitudes towards activities that individuals are unmotivated to undertake by embedding them in game-like environments. Gamification's effect on motivation is supported by theories from psychology and behavioral economics as well as game design know-how. Ultimately, Dr. Buzdugan's idea for a gamification-based approach built on the existing expertise within our research team, as gamification harnesses the power of many tools from behavioral science (e.g., incentives, commitments, reminders) in a context of fun. 
mHealth: How was the study carried out and what were the findings? What inspiration did it bring for future studies?

Prof. McCoy: The study published in $m$ Health focused on young gay, bisexual, and queer men in California, who experience a disproportionate burden of HIV and STIs. We developed and piloted an intervention to promote HIV and STI screening using concepts of gamification, which leverages theory and tools from behavioral science to motivate people to engage in a behavior in a context of fun.

We worked with YMSM and health care providers to develop the intervention which was named Stick To It. The program had four components: (I) recruitment (clinic-based and online); (II) online enrollment; (III) online activities; and (IV) 'real-world' activities at the clinic. For each activity in Stick To It, YMSM earned points through online activities that could be redeemed for a chance to win prizes during HIV screening and care visits at a local clinic.

After developing the intervention, we conducted a six-month pilot study of Stick To It with YMSM who lived in a zip code adjacent to one of the two study clinics in Oakland and Hollywood, California. We found that although the intervention was acceptable to study participants, engagement was modest. For example, about 1 in 5 YMSM completed at least one online activity, such as a quiz, in the subsequent 6 months after enrollment. In addition, points were redeemed in clinic by only $11 \%$ of the 166 study participants. Nevertheless, this level of engagement is similar to some other online health interventions and is not atypical from data from the private sector, which suggest that engagement with a mobile app is nearly always characterized by a pattern of early attrition after download.

Despite these challenges, men reported in interviews that they enjoyed the Stick To It program and that the inclusion of game elements was motivating. In addition, in a subset of men with medical record data, repeat HIV testing was higher in men who were in Stick To It compared to similar men who lived in the same zip codes and who received care at the same clinics before the intervention. These data indicate the promise of this approach for improving the health of YMSM and the need for future studies to refine and evaluate mHealth interventions using gamification.

\section{mHealth: Having been supported by the NIH funding, would you introduce us to a recent project that you are involved in?}

Prof. McCoy: Using tools from human-centered design and behavioral economics, our team is engaged in an NIHfunded project, "Ambassadors for Health", that focuses on adolescent girls and young women in Tanzania. There, as in many parts of the world, young women face the dual threats of HIV infection and unintended pregnancy that severely undermines their long-term wellbeing. However, health systems are often ill equipped to overcome the numerous barriers to health care services faced by young women.

In this project, our goal is to develop 'girl-friendly' drug shops (known as Accredited Drug Dispensing Outlets, or ADDOs, in Tanzania) as a venue where young women can access HIV prevention services and contraception. The motivation for this approach is the growing recognition that drug shops, which are ubiquitous in urban and rural settings in many parts of the world, can promote beneficial health behaviors, bridge gaps in health services, and mitigate health workforce shortages. At girl-friendly drug shops, we will distribute HIV self-test kits and contraception. We will measure whether there is demand for these products and whether drug shops are a suitable venue for their distribution. We will also provide linkages to local health care providers in case a young woman requires clinical evaluation (e.g., for HIV infection). Our long-term goal is to provide guidance about whether communitybased distribution of HIV testing at drug shops is an effective strategy for decreasing the incidence of HIV and unintended pregnancies among girls and young women. We started the project in mid-2018 and we should have the first set of results in 2020 .

\section{mHealtb: Did you come across any bottleneck throughout your career? How did you cope with it?}

Prof. McCoy: Young researchers face many challenges as they launch their careers, and one that I experienced early in my career was self-doubt. This affects many aspects of the scientific enterprise, from building collaborations, making bold decisions about research topics, and pursuing one's ideal life and career. For example, one of my dissertation papers was to describe and understand why people who know that they are living with HIV infection don't begin HIV primary care, a problem known as poor 'linkage to care'. At the time, despite the field's growing interest in behavioral aspects of HIV prevention and care, I was very self-conscious about the work-"Was it scholarly enough? Was it technical enough? Should I instead be working at the bench? Do the results matter?"

Little did I know that a decade later, the entire field of 
HIV prevention and care would be focused on poor linkage to care (the "second 90" in UNAIDS parlance), and even in 2018, some of the barriers described in my dissertation papers, like stigma and poverty, remain obstacles to global epidemic control. I wish I could tell my younger self to ignore the voice of self-doubt and instead feel confident in the fact that I was working on an important and relevant scientific problem. In that way, getting older has its benefits, because you become less worried about comparing yourself to others and instead focus more on charting a course of rigorous science that is professionally rewarding and important to the field.

mHealth: As an Associate Professor, what would be your advice to your students who would like to engage in your field of research?

Prof. McCoy: The most important advice is to recognize that there are many pathways to be successful in public health. I tell my graduate students to say yes to as many experiences as possible in order to fill up their 'toolbox', whether that means getting experience collecting data, working in a clinic, conducting research abroad, cleaning and analyzing data, writing reports, etc. Even if an opportunity is outside of one's substantive area of expertise (e.g., sexual health, cancer), there is likely still a lot to learn about process, methods, and/or leadership.

One persistent challenge is that there's not much emphasis on writing in STEM graduate programs, yet scientists in all disciplines need to be excellent communicators to be good at their jobs. So, any

doi: 10.21037/mhealth.2018.12.03

Cite this article as: $\mathrm{Li}$ B. Sandra I. McCoy: leveraging elements of games to improve sexual health. mHealth 2018;4:59. opportunities that come your way to refine your writing skills-whether that be ethical review board applications, grants, or manuscripts-will pay dividends in the future.

Lastly, I've benefitted from the advice and guidance of many mentors throughout my career and continue to do so today. Pay attention to the people around you who may be unexpected mentors-including those outside your field of interest-and cultivate those valuable relationships. Those individuals will likely be cheering you on throughout your entire career.

\section{Acknowledgements}

We would like to express our sincerest gratitude to Prof. Sandra I. McCoy for sharing her insights and opinions with us.

\section{Footnote}

Conflicts of Interest: The author has no conflicts of interest to declare.

\section{References}

1. HIV.gov. U.S. Statistics. Available online: https://www.hiv. gov/hiv-basics/overview/data-and-trends/statistics

2. McCoy SI, Buzdugan R, Grimball R, et al. Stick To It: pilot study results of an intervention using gamification to increase HIV screening among young men who have sex with men in California. Mhealth 2018;4:40.

(Science Editor: Brad Li, mhealth, editor@themhealth.org) 